CARDIOVASCULAR MEDICINE

\title{
Right ventricular functional recovery after acute myocardial infarction: relation with left ventricular function and interventricular septum motion. GISSI-3 echo substudy
}

\author{
B A Popescu, F Antonini-Canterin, P L Temporelli, P Giannuzzi, E Bosimini, F Gentile, A P Maggioni, \\ L Tavazzi, R Piazza, L Ascione, I Stoian, E Cervesato, A C Popescu, G L Nicolosi, for the GISSI-3 \\ Echo Substudy Investigators
}

Heart 2005;91:484-488. doi: 10.1136/hrt.2003.028050

See end of article for authors' affiliations

Correspondence to: Dr Bogdan A Popescu, Prof $\operatorname{Dr} C$ C lliescu Institute of Cardiovascular Diseases, Sos Fundeni 258, sector 2, 022328 Bucharest, Romania; abpope07@ $\mathrm{cmb}$.ro

Accepted 2 June 2004

\begin{abstract}
Objective: To evaluate the pattern of right ventricular (RV) functional recovery and its relation with left ventricular (LV) function and interventricular septal (IVS) motion in low risk patients after acute myocardial infarction (AMI).

Design and setting: Multicentre clinical trial carried out in 47 Italian coronary care units.

Patients: 500 patients from the GISSI (Gruppo Italiano per lo Studio della Sopravvivenza nell'Infarto Miocardico) - 3 echo substudy, who underwent serial echocardiograms 24-48 hours after symptom onset and at discharge, six weeks, and six months after AMI.

Results: Tricuspid annular plane systolic excursion (TAPSE) increased significantly during follow up (mean (SD) $1.79(0.46) \mathrm{cm}$ at $24-48$ hours to $1.92(0.46) \mathrm{cm}$ at six months, $\mathrm{p}<0.001)$ and the increase was already significant at discharge $(1.88(0.47) \mathrm{cm}, \mathrm{p}<0.001)$. LV ejection fraction (LVEF) was the best correlate of TAPSE at 24-48 hours $(r=0.15, p=0.001)$. TAPSE increased significantly in patients both with reduced $(<45 \%)$ and with preserved $(\geqslant 45 \%)$ LVEF, but the magnitude of increase was higher in patients with lower initial LVEF ( $p=0.001)$. Improvement in IVS wall motion score index (IVS-WMSI) was the only independent predictor of TAPSE changes during follow up $(r=-0.12, p=0.007)$.

Conclusions: In low risk patients after AMI, RV function recovered throughout six months of follow up and was already significant at discharge. TAPSE was significantly related to LVEF at 24-48 hours. The magnitude of RV functional recovery was higher in patients with lower initial LVEF. RV functional recovery is best related to IVS-WMSI improvement, suggesting that IVS motion has an important role in RV functional improvement in this setting.
\end{abstract}

M ost research on right ventricular (RV) function after acute myocardial infarction (AMI) has concentrated on the prognostic role of RV dysfunction in the long term outcome. This was mainly determined by assessing RV function in the acute phase of myocardial infarction and by clinical follow up thereafter. ${ }^{12}$ However, little is known about the pattern of RV functional recovery, its relation to global and regional left ventricular (LV) function, and the determinants of RV function change, as assessed by serial echocardiographic studies, in patients with low risk AMI.

We therefore examined the pattern of RV functional change and its correlations with global and regional LV function parameters at baseline and during six months' follow up in patients with low risk AMI. In addition, we sought to determine whether changes in RV function are different in patients with reduced, as opposed to preserved, LV ejection fraction (LVEF) and to identify the predictors of $\mathrm{RV}$ functional changes during follow up in this setting.

\section{METHODS}

Patients

The GISSI (Gruppo Italiano per lo Studio della Sopravvivenza nell'Infarto Miocardico) -3 echo substudy initially evaluated 878 patients among the 19394 patients randomly assigned to treatment within the GISSI-3 study. ${ }^{3}$ Patients were initially considered eligible if they had confirmed AMI as previously reported ${ }^{3}$ and echocardiogram recordings suitable for qualitative and quantitative analyses. The protocol required serial echocardiograms 24-48 hours (mean (SD) 36 (8) hours) after symptom onset and at hospital discharge (12 (5) days), six weeks (48 (9) days), and six months (194 (17) days) after AMI. ${ }^{4}$ The protocol was approved by the local ethics committee. Patients gave their informed consent.

\section{Echocardiographic study}

All the echocardiograms were submitted to the core laboratory at the research centre of the National Association of Hospital Cardiologists (Associazione Nazionale Medici Cardiologi Ospedalieri) in Florence for an assessment of quality and suitability for quantitative analysis. Scans were considered acceptable if they allowed visualisation of all myocardial segments from at least two complementary or orthogonal views and visual assessment of both endocardial motion and myocardial thickening. All parameters analysed were calculated as the mean of at least three measurements. For wall motion analysis a 16 segment model was used and each myocardial segment was scored according to the American Society of Echocardiography recommendations. ${ }^{5}$ Videotapes were analysed centrally by three expert investigators unaware of patients' clinical, ECG, or angiographic data, who assigned the wall motion score to each segment by consensus. Our interobserver and intraobserver

Abbreviations: AMI, acute myocardial infarction; BSA, body surface area; GISSI, Gruppo Italiano per lo Studio della Sopravvivenza nell'Infarto Miocardico; IVS, interventricular septum; LAA, left atrial maximum end systolic area; LV, left ventricular; LVEF, left ventricular ejection fraction; RV, right ventricular; TAPSE, tricuspid annular plane systolic excursion; WMSI, wall motion score index 


\begin{tabular}{|lc|}
\hline $\begin{array}{l}\text { Table } 1 \text { Baseline characteristics of the study population } \\
(\mathrm{n}=500)\end{array}$ \\
\hline Characteristic & Number $(\%)$ \\
\hline Men & $414(82.8 \%)$ \\
Age $>70$ years & $110(22.0 \%)$ \\
Site of infarction & \\
Anterior & $152(30.4 \%)$ \\
Inferoposterior & $186(37.2 \%)$ \\
Multiple locations & $15(3.0 \%)$ \\
Non-Q wave & $97(19.4 \%)$ \\
Undefined & $37(7.4 \%)$ \\
Not reported & $13(2.6 \%)$ \\
Killip class at admission & $431(86.4 \%)$ \\
1 & $64(12.8 \%)$ \\
2 & $4(0.8 \%)$ \\
3 & $62(12.5 \%)$ \\
Previous myocardial infarction & $173(35.7 \%)$ \\
Hypertension & $66(13.5 \%)$ \\
Diabetes & $351(70.8 \%)$ \\
Recommended treatments & $127(25.7 \%)$ \\
Thrombolysis & $432(87.3 \%)$ \\
Intravenous $\beta$ blockers & \\
Aspirin & \\
\hline &
\end{tabular}

reproducibility in wall motion score assessment were $89 \%$ and $93 \%$, respectively. ${ }^{6}$ Global LV wall motion score index (WMSI) was calculated by dividing the sum of scores of myocardial segments by the total number of evaluated segments. Sums of scores were calculated for the five interventricular septal (IVS) segments and the $11 \mathrm{LV}$ free wall segments. The WMSI for the IVS (IVS-WMSI) and for the LV free wall were then derived by dividing the respective sum of scores by the number of segments evaluated. Mitral regurgitation was assessed by colour Doppler flow mapping and graded as mild, moderate, or severe as previously described. ${ }^{7}$ Echocardiographic images were then digitised to obtain endocardial contours and LV cavity areas at end diastole and end systole from two apical orthogonal (four and two chamber) views. Indexed LV volumes and LVEF were determined as previously described. ${ }^{4}$ From the apical four chamber view left atrial maximum end systolic area (LAA) was measured by tracing the outline of the atrial endocardium. LAA was indexed to body surface area (BSA) $\left(\right.$ LAAi $=$ LAA $/$ BSA $\left.\left(\mathrm{cm}^{2} / \mathrm{m}^{2}\right)\right)$. For RV systolic functional assessment the tricuspid annular plane systolic excursion (TAPSE) was measured by two dimensional echocardiography from the apical four chamber view at the RV free wall level, as previously described. ${ }^{8}$ Timing of RV function changes was defined as "early" when it occurred during the inhospital phase from 24-48 hours to discharge and "late" when it occurred between discharge and six months. ${ }^{9}$ Overall changes of echocardiographic parameters were calculated as the difference between the respective values measured at six months and at 24-48 hours.

\section{Follow up}

The vital status at four years (dead or alive) was sought for all patients through the census office of their towns of residence by means of a prepaid return mail questionnaire. Information on death from any cause at the four year follow up was available for 469 (94\%) of the 500 patients, who formed the final study population.

\section{Statistical analysis}

Descriptive data are given as mean (SD). Differences between patients were assessed by unpaired $t$ testing and frequency of parameters by the $\chi^{2}$ test. Differences of echocardiographic measurements between groups and changes over time within each group (time effect) as well as any interaction (different trends over time between groups) were assessed by repeated measures analysis of variance. Linear regression analysis was used and correlation coefficients were calculated by Pearson's method. Stepwise multivariate linear regression analysis, with demographic, clinical, and echocardiographic variables included in the model, was performed to assess the independent predictors of TAPSE at 24-48 hours and of TAPSE changes. Variables included in the stepwise multivariate analysis were selected based on the best results of the univariate analyses (at the significance level of $\mathrm{p}<0.10$ ). Cardiac survival curves were computed with the KaplanMeier method. For this study the ability of TAPSE at 24-48 hours and of TAPSE changes to predict all cause mortality were assessed. Values of $p<0.05$ were considered significant.

\section{RESULTS}

\section{Clinical characteristics}

Patients with reinfarction (23 of $878 ; 2.6 \%$ ), myocardial revascularisation procedures $(\mathrm{n}=75 ; 8.5 \%)$, or who died during the six month echocardiographic follow up $(\mathrm{n}=36$; $4.1 \%$ ) and patients with incomplete echocardiographic follow up or images insufficient for quantitative analysis ( $\mathrm{n}=244$; $27.8 \%)$ were excluded from analysis. The remaining 500 patients (414 men, mean age (SD) 60.8 (11.8) years, range 22-90 years) who survived six months after AMI and underwent all four echocardiographic examinations constituted the final study population. Table 1 presents baseline characteristics of the study group. These characteristics were comparable with those of the general population of the GISSI-3 trial discharged alive and followed up with echocardiographic examinations at six months. ${ }^{10}$ Table 2 presents changes in echocardiographic parameters during follow up. The prevalence of significant mitral regurgitation (grade 2-3/3) did not change from 24-48 hours to six months: $7.1 \%, 10.4 \%$, $8.8 \%$, and $9.2 \%$, respectively $(p=0.58)$. Clinical event occurrence during follow up was low (table 3). During the four year follow up period, 40 patients died, with an estimated overall survival of $91 \%$.

\section{RV function at 24-48 hours}

Table 4 presents the correlations of TAPSE with the parameters at 24-48 hours. TAPSE was lower in patients with a previous myocardial infarction than in those with a first AMI (mean (SD) $1.67(0.47) \mathrm{cm} v 1.81(0.46) \mathrm{cm}$, $\mathrm{p}=0.02)$. There were no significant differences in TAPSE at 24-48 hours with respect to sex, infarct location, use of thrombolysis, heart failure occurrence at six months, or vital status at four years. At stepwise multivariate regression analysis LVEF was the only independent predictor of TAPSE at 24-48 hours $(r=0.15, \mathrm{p}=0.001)$.

\section{RV functional change during follow up}

There was a significant increase in TAPSE throughout follow up (fig 1), already present at discharge, reflecting early RV functional recovery (time effect $F=26.7, \mathrm{p}<0.001$ ). Late $\mathrm{RV}$ functional recovery was also significant (time effect $F=7.3, \mathrm{p}=0.007)$.

\section{Relation between RV and LV function at 24-48 hours and during follow up}

At 24-48 hours, TAPSE was significantly lower in patients with LVEF $<45 \%$ (group $\mathrm{A}, \mathrm{n}=161$ ) than in patients with LVEF $\geqslant 45 \%$ (group $\mathrm{B}, \mathrm{n}=339$ ) (mean (SD) 1.68 (0.43) $v$ $1.85(0.47) \mathrm{cm}, \mathrm{p}<0.001)$. TAPSE increased significantly in both groups during follow up, but the magnitude of increase was higher in group A (between subjects effect $F=11.1$, $p=0.001)$. TAPSE change was not different with respect to sex, infarct location, previous myocardial infarction, use of 
Table 2 Changes in echocardiographic parameters after AMI during six months' follow up $(n=500)$

\begin{tabular}{|c|c|c|c|c|c|}
\hline & $24-48$ hours & At discharge & 6 weeks & 6 months & $\begin{array}{l}\text { Time effect } \\
p \text { value }\end{array}$ \\
\hline EDVi $\left(\mathrm{ml} / \mathrm{m}^{2}\right)$ & 79.7 (19.2) & $83.9(22.6)$ & $85.9(24.9)$ & $86.7(26.1)$ & $<0.001$ \\
\hline ESVi $\left(\mathrm{ml} / \mathrm{m}^{2}\right)$ & $42.6(15.2)$ & $45.1(18.3)$ & $46.7(20.5)$ & $47.4(22.3)$ & $<0.001$ \\
\hline Global WMSI & $1.60(0.34)$ & $1.57(0.34)$ & $1.55(0.35)$ & $1.52(0.36)$ & $<0.001$ \\
\hline IVS-WMSI & 1.59 (0.53) & $1.56(0.53)$ & $1.53(0.51)$ & $1.49(0.51)$ & $<0.001$ \\
\hline LV-FWMSI & $1.61(0.34)$ & $1.58(0.34)$ & $1.56(0.35)$ & $1.54(0.36)$ & $<0.001$ \\
\hline LVEF (\%) & $47.2(7.3)$ & $47.2(8.2)$ & $46.9(8.2)$ & $46.9(8.6)$ & 0.25 \\
\hline TAPSE $(\mathrm{cm})$ & $1.79(0.46)$ & $1.88(0.47)$ & $1.89(0.48)$ & $1.92(0.46)$ & $<0.001$ \\
\hline LAAi $\left(\mathrm{cm}^{2} / \mathrm{m}^{2}\right)$ & $9.49(2.00)$ & $9.68(2.14)$ & $9.73(2.01)$ & $9.80(2.03)$ & 0.001 \\
\hline
\end{tabular}

Data are mean (SD).

AMI, acute myocardial infarction; EDVi, end diastolic volume index; ESVi, end systolic volume index; IVS, interventricular septum; LAAi, left atrial area index; LVEF, left ventricular ejection fraction; LV-FWMSI, left ventricular free wall motion score index; TAPSE, tricuspid annular plane systolic excursion; WMSI, wall motion score index.

thrombolysis, heart failure occurrence at six months, or four year vital status. Table 5 presents the correlations of overall TAPSE changes (six months - 24-48 hours) with the parameters. At stepwise multivariate regression analysis IVSWMSI changes emerged as the only independent predictor of TAPSE changes during follow up $(r=-0.12, \mathrm{p}=0.007)$.

\section{DISCUSSION}

\section{RV functional recovery}

That RV function recovers soon and to a great extent after an ischaemic insult is a long standing concept, proved both experimentally and clinically. Global RV performance recovers within days, regardless of the patency status of the infarct related artery. ${ }^{11}$ Even in chronic right coronary artery occlusion global RV performance improves greatly as early as postinfarction day 5, despite persistent severe RV free wall dysfunction. ${ }^{12}$ Reperfusion facilitates recovery of RV function and minimises the extent of infarction even after prolonged ischaemia. ${ }^{13}$ Serial echocardiograms recorded in 77 patients after infarction with three month follow up showed rapid normalisation of overall RV function (assessed by the myocardial performance index). ${ }^{14}$

Our study confirms in a larger population RV systolic functional recovery early after AMI and in addition shows that RV function continues to improve up to six months after infarction. We also confirm that RV function recovers to a greater extent than LV function. ${ }^{14}{ }^{15}$ Indeed, in the current study systolic RV function improved throughout follow up, whereas global LV systolic function remained essentially unchanged.

In this study TAPSE at 24-48 hours and TAPSE changes during follow up did not predict heart failure at six months or survival at four years. Exclusion of patients with adverse events during the six month echocardiographic follow up, the low mortality rate (no deaths at six months by design, four year mortality $<9 \%$ ) in this selected low risk population

Table 3 Clinical events recorded during follow up in the study population $(n=500)$

\begin{tabular}{lc}
\hline Time of event & Number (\%) \\
\hline In-hospital events & $30(6 \%)$ \\
AF or atrial flutter & $7(1.4 \%)$ \\
Sustained VT & $23(4.6 \%)$ \\
2nd or 3rd degree AV block & $1(0.2 \%)$ \\
Stroke & $110(22 \%)$ \\
Heart failure after discharge & $116(23.2 \%)$ \\
At 6 weeks & \\
At 6 months & \\
\hline AF, atrial fibrillation; AV, atrioventricular; VT, ventricular tachycardia.
\end{tabular}

with preserved LV ejection fraction, and our use of total mortality as the sole long term outcome event may account for this result. A more detailed analysis of survival determinants in this trial has been recently published. ${ }^{16}$

\section{RV functional recovery: the relation with LV function and IVS motion}

Moller and colleagues ${ }^{14}$ showed that in AMI, RV function correlated significantly with LV function; they also found weak correlations of RV myocardial performance index with global LV WMSI. The present study showed that in this low risk population RV function was related to LV function at 2448 hours. We also showed that during follow up RV function recovered in patients both with reduced and preserved LVEF, but the magnitude of change was higher in patients with lower initial LVEF.

The main finding of this study is the relation of follow up RV functional changes with global and regional LV functional changes and in particular with IVS-WMSI changes. Although at univariate analysis both LVEF changes and IVS-WMSI changes were significantly related to TAPSE changes, the only independent predictor of TAPSE changes at multivariate analysis was not global LVEF improvement but IVS-WMSI improvement. This suggests that the main mechanism of RV functional improvement in this setting is IVS motion itself.

This finding confirms the role of IVS motion in RV performance and is concordant with previous experimental studies showing that RV performance is dependent on LV septal contractile contributions transmitted through systolic ventricular interaction. ${ }^{17}{ }^{18}$ In another study, ${ }^{19}$ the presence or

Table 4 Univariate and multivariate correlates of TAPSE at 24-48 hours after symptom onset $(n=500)$

\begin{tabular}{|c|c|c|c|c|}
\hline \multirow[b]{2}{*}{ Variable } & \multicolumn{2}{|c|}{ Univariate analysis } & \multicolumn{2}{|c|}{ Multivariate analysis } \\
\hline & $R$ coefficient & p Value & $\begin{array}{l}\text { Multi-R } \\
\text { coefficient }\end{array}$ & p Value \\
\hline Age & 0.05 & 0.28 & & \\
\hline LVEF & 0.15 & 0.001 & 0.15 & 0.001 \\
\hline EDVi & 0.03 & 0.49 & & \\
\hline ESVi & 0.03 & 0.44 & & \\
\hline Global WMSI & 0.03 & 0.49 & & \\
\hline IVS-WMSI & 0.08 & 0.07 & & \\
\hline LV-FWMSI & 0.01 & 0.78 & & \\
\hline$E$ & 0.08 & 0.091 & & \\
\hline Edt & 0.04 & 0.33 & & \\
\hline LAAi & 0.02 & 0.61 & & \\
\hline Peak CK release & 0.04 & 0.41 & & \\
\hline
\end{tabular}




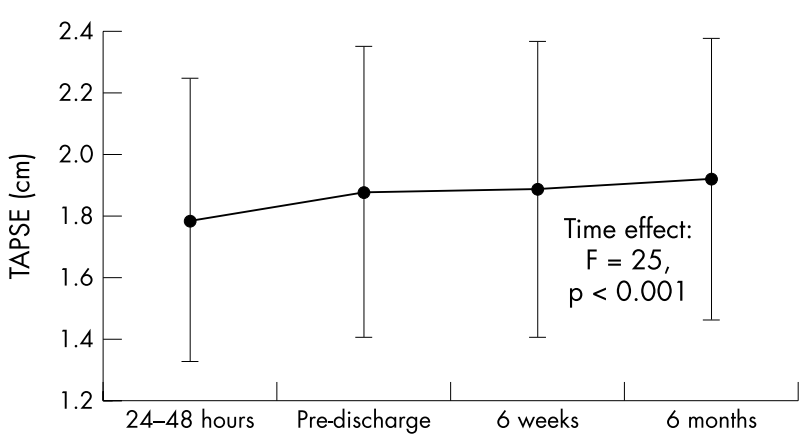

Figure 1 Changes of tricuspid annular plane systolic excursion (TAPSE) from the acute infarction to six months.

absence of IVS involvement was a significant determinant of $\mathrm{RV}$ response to exercise in patients with recent myocardial infarction, suggesting that IVS contraction may be important for the maintenance of RV function in patients after infarction with impaired LV function. Our study showed that even in patients at low risk after AMI, improvement in $\mathrm{RV}$ function is related to improvement in IVS wall motion abnormalities.

Although the IVS has long been regarded as a functional part of the LV, it has lately been shown that the IVS actually contributes to the systolic function of both ventricles. An experimental study showed that RV function was impaired as much with septal ligation as with right coronary artery ligation, ${ }^{20}$ showing that ischaemic injury to either the IVS or the RV free wall alters RV function and suggesting that both regions play a significant part in maintaining RV performance. The important role of the IVS in RV performance was later confirmed in patients by means of biventricular angiography, with thickening of the IVS being shown to contribute to the systolic contraction of both ventricles. ${ }^{21}$

To our knowledge, this study is the first showing the relation between RV functional recovery and improvement in IVS wall motion abnormalities in a large population of patients after an infarction within a randomised, multicentre trial, by serial echocardiograms. This offers important pathophysiological insights into RV functional recovery after AMI.

\section{Limitations}

Echocardiography was first performed at 36 (8) hours from symptom onset, when it is possible that some RV function had already been recovered. Nevertheless, since our purpose was to assess not RV function at one moment in time but recovery of RV function serially, the possibility of partial RV functional recovery does not alter the conclusions of the study. RV systolic function was assessed only by measuring TAPSE. However, accurate methods such as radionuclide ventriculography and magnetic resonance imaging are time consuming, relatively expensive, and cannot be used at the bedside. The complex shape of the RV precludes an accurate volumetric assessment by echocardiography and poor endocardial definition usually limits calculation of RV fractional area change. TAPSE measurement is devoid of geometrical assumptions or traceable endocardial outlines, is easy to measure, reproducible, and feasible, ${ }^{22}$ has a very good correlation with RV ejection fraction, ${ }^{823}$ and is recognised as an important tool for assessing RV systolic function. ${ }^{23} 24$ Although the absolute changes of TAPSE were small, they were highly significant. The small magnitude of TAPSE changes can be explained by the small mean infarct size and lower severity of disease in this low risk population. The validity of our echocardiographic measurements in this study was ascertained by expert analysis in the core laboratory, with a low variability. ${ }^{46}$
Table 5 Univariate and multivariate correlates of TAPSE changes from $24-48$ hours after symptom onset to six months $(n=500)$

\begin{tabular}{|c|c|c|c|c|}
\hline \multirow[b]{2}{*}{ Variable } & \multicolumn{2}{|c|}{ Univariate analysis } & \multicolumn{2}{|c|}{ Multivariate analysis } \\
\hline & $R$ coefficient & p Value & $\begin{array}{l}\text { Multi-R } \\
\text { coefficient }\end{array}$ & p Value \\
\hline Age & 0.01 & 0.78 & & \\
\hline \multicolumn{5}{|c|}{ Variables at $24-48$ hours after symptom onset } \\
\hline LVEF & 0.06 & 0.16 & & \\
\hline $\mathrm{E}$ & 0.04 & 0.35 & & \\
\hline Edt & 0.03 & 0.52 & & \\
\hline Global WMSI & 0.02 & 0.69 & & \\
\hline IVS WMSI & 0.03 & 0.53 & & \\
\hline LV-FWMSI & 0.006 & 0.90 & & \\
\hline Peak CK & 0.01 & 0.75 & & \\
\hline \multicolumn{5}{|c|}{ Change from $24-48$ hours after symptom onset to 6 weeks } \\
\hline LVEF & 0.12 & 0.008 & & \\
\hline EDVi & 0.06 & 0.15 & & \\
\hline ESVi & 0.003 & 0.94 & & \\
\hline$M R$ & 0.004 & 0.93 & & \\
\hline LAAi & 0.05 & 0.32 & & \\
\hline Global WMSI & -0.11 & 0.013 & & \\
\hline IVS-WMSI & -0.12 & 0.005 & -0.12 & 0.007 \\
\hline LV-FWMSI & -0.08 & 0.086 & & \\
\hline
\end{tabular}

\section{Conclusions}

This study shows that in low risk patients after AMI, RV function recovers throughout six month follow up and is already significant at discharge. TAPSE is significantly related to LVEF at 24-48 hours. RV function recovers in patients both with reduced and with preserved LVEF, but the magnitude of change is higher in patients with lower initial LVEF. RV function change is best related to IVS-WMSI improvement, suggesting that IVS motion has an important role in RV function improvement in this setting.

\section{ACKNOWLEDGEMENTS}

Drs B A Popescu and A C Popescu were supported by Research Fellowships of the Association for Research in Cardiology, Pordenone, Italy.

\section{Authors' affiliations}

B A Popescu, F Antonini-Canterin, R Piazza, L Ascione, I Stoian, E Cervesato, A C Popescu, G L Nicolosi, Ospedale Civile, A R C, Pordenone, Italy

P L Temporelli, P Giannuzzi, E Bosimini, The Fondazione Maugeri, Istituto di Ricovero e Cura a Carattere Scientifico, Veruno, Italy F Gentile, Ospedale Bassini, Cinisello Balsamo, Italy

A P Maggioni, Centro Studi Associazione Nazionale Medici Cardiologi Ospedalieri, Florence, Italy

L Tavazzi, Ospedale S Matteo, Pavia, Italy

GISSI is endorsed by the Associazione Nazionale Medici Cardiologi Ospedalieri (ANMCO, Italy) and the Istituto di Ricerche Farmacologiche "Mario Negri", Milan, Italy.

For list of investigators see reference 7 (appendix).

\section{REFERENCES}

1 Samad BA, Alam M, Jensen-Urstad K. Prognostic impact of right ventricular involvement as assessed by tricuspid annular motion in patients with acute myocardial infarction. Am J Cardiol 2002;90:778-81.

2 Zornoff LAM, Skali H, Pfeffer MA, et al. Right ventricular dysfunction and risk of heart failure and mortality after myocardial infarction. $J \mathrm{Am}$ Coll Cardiol 2002;39:1450-5

3 GISSI-3 Investigators. GISSI-3: effects of lisinopril and transdermal glyceryl trinitrate singly and together on 6-week mortality and ventricular function after acute myocardial infarction, Gruppo Italiano per lo Studio della Sopravvivenza nell'Infarto Miocardico. Lancet 1994;343:1115-22.

4 Bosimini E, Giannuzzi P, Temporelli PL, et al. Electrocardiographic evolutionary changes and left ventricular remodeling after acute myocardial infarction: results of the GISSI-3 echo substudy. J Am Coll Cardiol 2000;35:127-35 
5 Schiller NB, Shah PM, Crawford M, et al. Recommendations for quantitation of left ventricle by two-dimensional echocardiography. American Society of Echocardiography committee on standards, subcommittee on quantitation of two-dimensional echocardiograms. J Am Soc Echocardiogr 1989;2:358-67.

6 Badano L, Stoian J, Cervesato E, et al, for the GISSI-3 Echo Substudy Investigators. Reproducibility of wall motion score and its correlation with left ventricular ejection fraction in patients with acute myocardial infarction. Am J Cardiol 1996:78:855-8.

7 Ascione L, Antonini-Canterin F, Macor F, et al. Relation between early mitral regurgitation and left ventricular thrombus formation after acute myocardial infarction: results of the GISSI-3 echo substudy. Heart 2002;88:131-6.

$8 \mathrm{Kaul} \mathrm{S}$, Tei C, Hopkins JM, et al. Assessment of right ventricular function using two-dimensional echocardiography. Am Heart J 1984;107:526-31.

9 Giannuzzi P, Temporelli PL, Bosimini E, et al. Heterogeneity of left ventricular remodeling after acute myocardial infarction: results of the Gruppo Italiano per lo Studio della Sopravvivenza nell'Infarto Miocardico-3 echo substudy. Am Heart J 2001;141:131-8.

10 Nicolosi GL, Latini R, Marino P, et al. Gruppo Italiano per lo Studio della Sopravvivenza nell'Infarto Miocardico: the prognostic value of predischarge quantitative two-dimensional echocardiographic measurements and the effects of early lisinopril treatment on left ventricular structure and function after acute myocardial infarction in the GISSI-3 Trial. Eur Heart J 1996;17:1646-56.

11 Steele P, Kirch D, Ellis J, et al. Prompt return to normal of depressed right ventricular ejection fraction in acute inferior infarction. Br Heart $J$ 1977;39:1319-23.

12 Laster SB, Shelton TJ, Barzilai B, et al. Determinants of the recovery of right ventricular performance following experimental chronic right coronary artery occlusion. Circulation 1993:88:696-708.

13 Laster SB, Ohnishi Y, Saffitz JE, et al. Effects of reperfusion on ischemic right ventricular dysfunction: disparate mechanisms of benefit related to duration of ischemia. Circulation 1994;90:1398-409.
14 Moller JE, Sondergaard E, Poulsen SH, et al. Serial Doppler echocardiographic assessment of left and right ventricular performance after a first myocardial infarction. J Am Soc Echocardiogr 2001;14:249-55.

15 Bowers TR, O'Neill WW, Grines $C$, et al. Effect of reperfusion on biventricular function and survival after right ventricular infarction. N Engl J Med 1998:338:933-40

16 Temporelli PL, Giannuzzi P, Nicolosi GL, et al. Doppler-derived mitral deceleration time as a strong prognostic marker of left ventricular remodeling and survival after acute myocardial infarction: results of the GISSI-3 echo substudy. J Am Coll Cardiol 2004:43:1646-53.

17 Goldstein JA, Tweddell JS, Barzilai B, et al. Importance of left ventricular function and systolic ventricular interaction to right ventricular performance during acute right heart ischemia. J Am Coll Cardiol 1992;19:704-11.

18 Sharkey SW, Shelley W, Carlyle PF, et al. M-mode and two-dimensional echocardiographic analysis of the septum in experimental right ventricular infarction: correlation with hemodynamic alterations. Am Heart J 1985;110:1210-8

19 Nakamura S, Iwasaka T, Kimura Y, et al. Right ventricular ejection fraction during exercise in patients with recent myocardial infarction: effect of the interventricular septum. Am Heart J 1994;127:49-55.

20 Fixler DE, Monroe GA, Wheeler JM. Hemodynamic alterations during septal or right ventricular ischemia in dogs. Am Heart J 1977;93:210-5.

21 Banka VS, Agarwal JB, Bodenheimer MM, et al. Interventricular septal motion: biventricular angiographic assessment of its relative contribution to left and right ventricular contraction. Circulation 1981;64:992-6.

22 Hammarstrom E, Wranne B, Pinto FJ, et al. Tricuspid annular motion. J Am Soc Echocardiogr 1991;4:131-9.

23 Ueti OM, Camargo EE, de A Ueti A, et al. Assessment of right ventricular function with Doppler echocardiographic indices derived from tricuspid annular motion: comparison with radionuclide angiography. Heart 2002;88:244-8.

24 Bleasdale RA, Frenneaux MP. Prognostic importance of right ventricular dysfunction. Heart 2002;88:323-4.

\section{IMAGES IN CARDIOLOGY}

\section{Subacute stent thrombosis successfully treated with thrombolysis and glycoprotein Ilb/Illa inhibition}

A 35 year old man underwent elective angioplasty for a severe stenosis at the ostium of the left anterior descending artery (LAD) involving the distal left main stem (LMS) (panel A). Abciximab was administered and a $3.5 \times 24 \mathrm{~mm}$ Taxus stent (Boston Scientific, USA) was deployed in the proximal LAD extending into the distal LMS, bridging the circumflex ostium. Flow was now compromised in the circumflex and a second stent $(2.5 \times 16 \mathrm{~mm}$ Taxus) was deployed at the circumflex ostium in a " $\mathrm{T}$ " configuration. Further dilatations were performed to both stents and a good final angiographic result was obtained (panel B).

Against medical advice the patient stopped clopidogrel at 10 days. Thirty days postangioplasty he presented to the emergency room at a referring hospital with acute chest pain. ECG showed anterior ST depression and lateral ST elevation. The patient was thrombolysed with tenecteplase and transferred for urgent coronary angiography. The LAD/LMS stent was widely patent, however, the circumflex stent contained thrombus (panel C) with TIMI 3 flow in the circumflex. As he was pain-free and stable a decision was made to attempt medical treatment. Tirofiban was administered for 48 hours. Angiography at 48 hours showed the thrombus in the circumflex had resolved (panel D). His ECG returned to normal and he suffered no further chest pain.

Stent thrombosis is believed to have occurred because of premature discontinuation of clopidogrel and was successfully


managed medically with a combination of thrombolysis and glycoprotein IIb/IIIa inhibition. At four months follow up the patient was free of angina. 